\title{
Análisis de la partícula japonesa ni en oraciones que expresan estados locativos
}

\section{Analysis of the Japanese particle ni in sentences that express locative states}

\author{
Andrea Rodríguez Bravo \\ Universidad Nacional Mayor de San Marcos, \\ Lima-Perú \\ andrea.rodriguez4@unmsm.edu.pe \\ https://orcid.org/oooo-0002-2282-8242
}

\author{
Claudia Ximena Garay Ramírez \\ Universidad Nacional Mayor de San Marcos, \\ Lima-Perú \\ claudia.garay@unmsm.edu.pe \\ https://orcid.org/oooo-00o2-2999-8914
}

\begin{abstract}
RESUMEN
Este artículo trata sobre la variación de la partícula ni en oraciones que refieren estados locativos en la lengua japonesa. Se presentan los resultados del análisis de las partículas: wa, ga, y no; así como también la alternancia de uso de los verbos arimasu e imasu. El corpus del presente trabajo se divide en oraciones excesivas e inesivas, cada una con su respectiva formalización siguiendo la propuesta de Moreno Cabrera (2003). Finalmente, se demuestra lo postulado por Langacker (1987, como se citó en Moreno, 2003), quien postula que los adjetivos, adverbios y preposiciones determinan la relación de atemporalidad y, aunque el tiempo se presente no resulta relevante. De esta manera, se resalta el papel de la locatividad. En las oraciones analizadas, se demuestra que los adverbios determinan las oraciones locativas.
\end{abstract}

Palabras clave: semántica formal; lengua japonesa; estados locativos; partícula ni

\begin{abstract}
This article deals with the variation of the particle $n i$ in sentences that refer to locative states in the Japanese language. The results of the analysis of the particles are presented: $w a, g a$, and no; as well as the alternation of use of the verbs arimasu and imasu. The corpus of the present work is divided into excessive and ineffective sentences, each one with its respective formalization following the proposal of Moreno Cabrera (2003). Finally, the postulated by Langacker (1987, as cited in Moreno, 2003) is demonstrated, who postulates that adjectives, adverbs and prepositions determine the relationship of timelessness and, although time is present, it is not relevant. In this way, the role of locativity is highlighted. In the analyzed sentences, it is shown that adverbs determine locative sentences.
\end{abstract}

Keywords: formal semantics; japanese language; locative states; particles

Recibido: I8-05-202I

Aprobado: 15-06-202I

Publicado: I9-I2-202I 
Análisis de la partícula japonesa ni en oraciones que expresan estados locativos Andrea Rodríguez Bravo y Claudia Ximena Garay Ramírez

\section{Introducción}

La lengua japonesa se caracteriza por el orden Sujeto + Objeto + Verbo, a diferencia del español que presenta un orden de Sujeto + Verbo + Objeto (Cardona, 2000). Además, presenta partículas en frases y oraciones. Las partículas son palabras auxiliares que se utilizan para indicar las funciones de sujeto, complemento directo, complemento circunstancial, entre otros. Estas no guardan correspondencia en el español, como por ejemplo las partículas de función semántica y de función estructural.

Considerando la polivalencia de la partícula - $n i$, el objetivo general de este trabajo es analizar su significado y función en oraciones que refieren estados en la lengua japonesa. Asimismo, se pretende explicar su relación con otras partículas también utilizadas en este tipo de oraciones, así como la alternancia de los verbos aru e iru.

La partícula -ni puede ser estructural (sin significado) o presentar carga semántica (temporal, espacial y existencia). Por ello, nos preguntamos qué determina la presencia y variación de esta en las oraciones locativas. Cabe mencionar que no se pretende establecer una generalización, sino solo brindar un acercamiento al comportamiento variable de las partículas en esta lengua.

\section{Marco referencial}

\subsection{La semántica formal}

El lenguaje formal es una herramienta de análisis que se representa las relaciones entre entidades y propiedades que forman parte de los modelos. En otras palabras, este tipo de lenguajes abstraen los contenidos de los enunciados. Así, el lenguaje natural es fundamentalmente ambiguo, mientras que el lenguaje formal es esencialmente preciso.

Según Varona (2020) la semántica formal se sirve de las técnicas lógicas de los lenguajes formales. La tarea de la Semántica formal es construir sistemas lógicos capaces de representar formalmente las expresiones del lenguaje natural. Según Moreno Cabrera (2003), la semántica no nos entrega el contenido conceptual de las expresiones, sino que ella es el enlace formal entre la sintaxis y dicho contenido.

Los sucesos se definen y estructuran unos a partir de otros en las oraciones simples, y pueden ser de tres tipos: estados, procesos o acciones. El interés de la presente investigación recae sobre las oraciones que refieren estados. Un estado 
Análisis de la partícula japonesa ni en oraciones que expresan estados locativos Andrea Rodríguez Bravo y Claudia Ximena Garay Ramírez

es concebido como un predicado que relaciona una entidad con un lugar o con una propiedad. En el primer caso el estado es locativo, por ejemplo: David está en Madrid, y en el segundo, atributivo, por ejemplo: Juan es alto. Según Langacker (citado por Moreno Cabrera, 2003), ambos tipos de estados denotan una relación de atemporalidad; de esta manera, la relación no está determinada en base al tiempo en el que ocurren estos sucesos. Además, los adjetivos adverbios y preposiciones denotan relaciones atemporales.

\section{Materiales y métodos}

La presente investigación es de tipo descriptivo-explicativo. Se emplea el marco teórico de la semántica formal. El corpus incluye solo oraciones simples que refieren estados locativos, de esta manera se presentan las oraciones en japonés, la traducción literal (el significado de cada palabra y partícula en español), la traducción al español y la formalización de este tipo de oraciones según la propuesta de Moreno Cabrera (2003).

El método empleado para la recolección del corpus consistió en entrevistas a dos hablantes bilingües japonés-español (uno nativo y un no nativo) residentes en Japón. En las entrevistas, primero se mostró imágenes al hablante nativo con el fin de evocar la elicitación de las oraciones que refieran a estados locativos, preguntando por el lugar o posición en el que estaban los objetos o personas mostrados. Posteriormente, el hablante no nativo contribuyó con la traducción de las oraciones.

\section{Análisis}

\subsection{Localizaciones excesivas}

En primer lugar, describiremos las oraciones excesivas, las cuales tienen como característica que un individuo A se localiza cerca o al lado sin tener contacto con un individuo B.

En (1) se presenta una relación de localización, entre las entidades on'nanoko ('niña') y teeburu ('mesa'). Específicamente se presenta una relación subesiva, puesto que la niña se posiciona shita («debajo») de teeburu (mesa). En la oración aparece el verbo imasu porque on'nanoko (niña) es un ser con voluntad. Además, se presenta la partícula wa encargada de marcar el sujeto de la oración on'nanoko (niña). Otra partícula con carga semántica que se presenta es no la cual expresa 
Análisis de la partícula japonesa ni en oraciones que expresan estados locativos Andrea Rodríguez Bravo y Claudia Ximena Garay Ramírez

que existe una relación entre on'nanoko (niña) y teeburu (mesa). En cuanto a la partícula ni, esta se presenta entre el adverbio shita (abajo) y el verbo imasu (estar).

(1)

$\begin{array}{lcccccc}\text { On'nanoko } & \text { wa } & \text { tēburu } & \text { no } & \text { shita } & \text { ni } & \text { imasu } \\ \text { niña/mujer } & \text { PART.TEMA } & \text { mesa } & \text { PART-REL } & \text { abajo } & \text { PARC.EST } & \text { v.estar } \\ & & & & & & \end{array}$

«La niña está debajo de la mesa»

\section{Formalización}

De acuerdo con lo establecido en la semántica formal, emplearemos el símbolo sigma para representar la relación atributiva de las entidades con el lugar o propiedad. De modo que la oración 1 se representa del siguiente modo: $\Sigma^{\text {shita }}$ (On'nanoko, teeburu), donde shita es un adverbio que expresa una localización subesiva.

\section{Gráfico 1. Diagrama arbóreo de la estructura semántica del estado locativo en la oración On'nanoko wa tēburu no shita ni imasu}

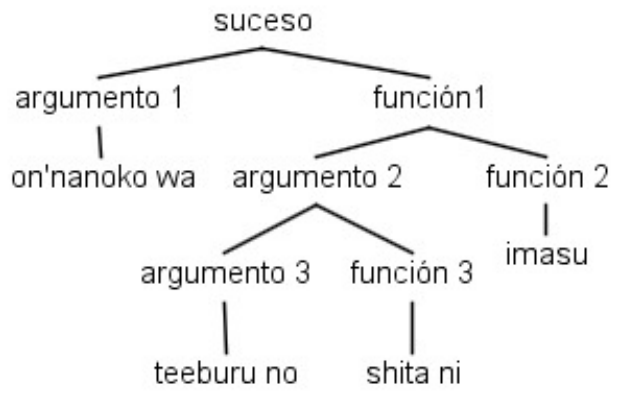

Como se observa en el gráfico 1, la partícula ni acompaña al adverbio shita (abajo) no aporta un significado, por lo tanto, cumple solo una función estructural, ya que se presenta de manera anterior al verbo imasu (estar).

En (2) se presenta una relación de localización, entre las entidades bōru (pelota) y teeburu (mesa). Específicamente se presenta una relación subesiva, puesto que la pelota se posiciona shita («debajo») de teeburu (mesa). En la oración aparece el verbo arimasu porque bōru (pelota) es un ser sin voluntad. Además, se presenta

184 Lengua y Sociedad. Revista de Lingüística Teórica y Aplicada 
Análisis de la partícula japonesa ni en oraciones que expresan estados locativos Andrea Rodríguez Bravo y Claudia Ximena Garay Ramírez

la partícula wa encargada de marcar el sujeto de la oración bōru (pelota). Otra partícula con carga semántica que se presenta es no la cual expresa que existe una relación entre bōru (pelota) y teeburu (mesa). En cuanto a la partícula $n i$, esta se presenta entre el adverbio shita (abajo) y el verbo arimasu (estar).

(2)

$\begin{array}{lcccccc}\text { Bōru } & w a & \text { tēburu } & n o & \text { shita } & \boldsymbol{n i} & \text { arimasu } \\ \text { pelota } & \text { PART.TEMA } & \text { mesa } & \text { PARC.REL } & \text { abajo } & \text { PARC.EST } & \text { verb.estar }\end{array}$

[-voluntad]

«La pelota está debajo de la mesa»

\section{Formalización}

De acuerdo con lo establecido en la semántica formal, emplearemos el símbolo sigma para representar la relación atributiva de las entidades con el lugar o propiedad. De modo que la oración 2 se representa del siguiente modo: $\sum^{\text {shita }}$ (Booru, teeburu), donde shita es un adverbio que expresa una localización subesiva.

Gráfico 2. Diagrama arbóreo de la estructura semántica del estado locativo en la oración Bōru wa tēburu no shita ni arimasu

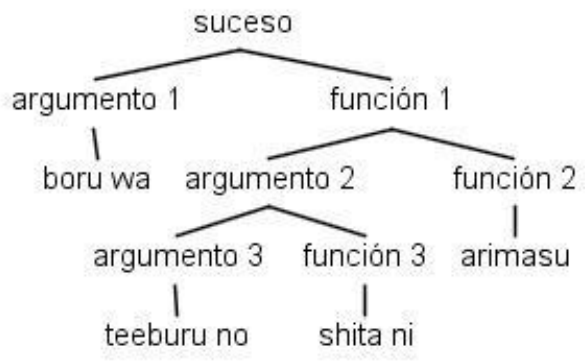

Como se observa en el gráfico 2, la partícula ni acompaña al adverbio shita (abajo) no aporta un significado, por lo tanto, cumple solo una función estructural, ya que se presenta de manera anterior al verbo arimasu (estar).

En (3) se presenta una relación de localización, entre las entidades ie (casa) y $k i$ (arbol). Específicamente se presenta una relación destral, puesto que la casa se 
Análisis de la partícula japonesa ni en oraciones que expresan estados locativos Andrea Rodríguez Bravo y Claudia Ximena Garay Ramírez

posiciona migigawa (a la derecha) del $k i$ (arbol). En la oración aparece el verbo arimasu porque ie (casa) es un ser sin voluntad. Además, se presenta la partícula ga esta cumple una función meramente estructural ya que no aporta algún significado en la oración. En cuanto a la partícula ni, esta se presenta entre el adverbio migigawa (a la derecha) y el verbo arimasu (haber).

(3)

$\begin{array}{lcccccc}\text { Ie } & \text { no } & \text { migigawa } & n \boldsymbol{n i} & k i & g a & \text { arimasu } \\ \text { casa } & \text { PART.REL } & \text { derecha } & \text { PERM.TEMP } & \text { árbol } & \text { PART.EST } & \text { verb.haber }\end{array}$

[-voluntad]

«Del lado derecho de la casa hay un árbol»

\section{Formalización}

De acuerdo con lo establecido en la semántica formal, emplearemos el símbolo sigma para representar la relación atributiva de las entidades con el lugar o propiedad. De modo que la oración 3 se representa del siguiente modo: $\sum^{\text {migigawa }}$ (Ie, ki) donde migigawa es un adverbio que expresa una localización destral.

\section{Gráfico 3. Diagrama arbóreo de la estructura semántica del estado locativo en la oración Ie no migigawa ni ki ga arimasu}

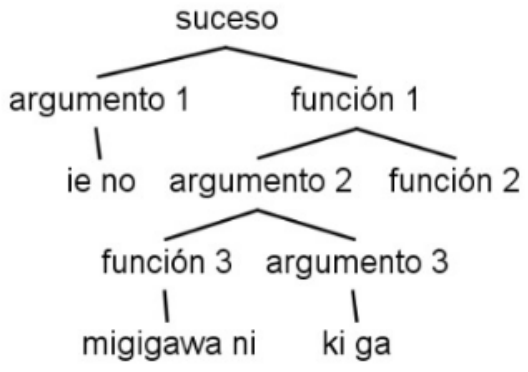

Como se observa en el gráfico 3, la partícula ni acompaña al adverbio migigawa (a la derecha) aporta un significado, por lo tanto, cumple solo una función semántica, ya que se presenta de manera anterior al sustantivo ki (árbol).

En (4) se presenta una relación de localización, entre las entidades inu (perro) y tabemono (comida). Específicamente se presenta una relación adhesiva, puesto 
Análisis de la partícula japonesa ni en oraciones que expresan estados locativos Andrea Rodríguez Bravo y Claudia Ximena Garay Ramírez

que el perro se posiciona chikaku (cerca) de tabemono (comida). En la oración aparece el verbo imasu porque inu (perro) es un ser con voluntad. Además, se presenta la partícula wa encargada de marcar el sujeto de la oración inu (perro). Otra partícula con carga semántica que se presenta es no la cual expresa que existe una relación entre inu (perro) y tabemono (comida). En cuanto a la partícula $n i$, esta se presenta entre el adverbio chikaku (cerca) y el verbo imasu (estar)

(4)

$\begin{aligned} & \text { Inu wa } \\ & \text { perro PART.TEMA }\end{aligned}$ comida
[+voluntad $]$
«El perro está cerca de su comida»

\section{Formalización}

De acuerdo con lo establecido en la semántica formal, emplearemos el símbolo sigma para representar la relación atributiva de las entidades con el lugar o propiedad. De modo que la oración 4 se representa del siguiente modo: $\sum$ chikaku (Ie, ki) donde chikaku es un adverbio que expresa una localización adhesiva.

\section{Gráfico 4. Diagrama arbóreo de la estructura semántica del estado locativo en la oración Inu w|a tabemono no chikaku ni imasu}

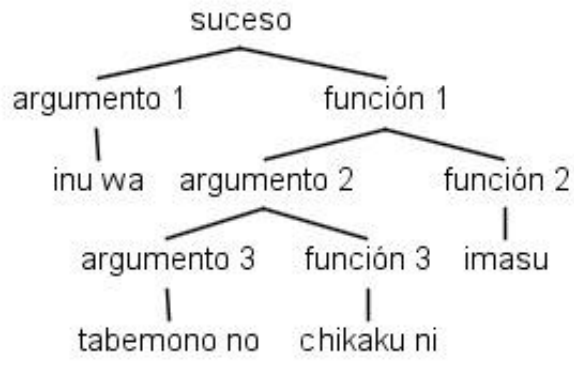

Como se observa en el gráfico 4, la partícula ni acompaña al adverbio chikaku (cerca) no aporta un significado, por lo tanto, cumple solo una función estructural, ya que se presenta de manera anterior al verbo imasu (estar). 
Análisis de la partícula japonesa ni en oraciones que expresan estados locativos Andrea Rodríguez Bravo y Claudia Ximena Garay Ramírez

\subsection{Localizaciones inesivas}

En segundo lugar, describiremos las oraciones excesivas, las cuales tienen como característica que un individuo A se localiza cerca o al lado de un individuo B, manteniendo contacto.

En (5) se presenta una relación de localización, entre las entidades kodomo (niño) y hako (caja). Específicamente se presenta una relación interior, puesto que el niño se posiciona naka (dentro) de hako (caja). En la oración aparece el verbo imasu porque kodomo (niño) es un ser con voluntad. Además, se presenta la partícula wa encargada de marcar el sujeto de la oración kodomo (niño). Otra partícula con carga semántica que se presenta es no la cual expresa que existe una relación entre kodomo (niño) y hako (caja). En cuanto a la partícula ni, esta se presenta entre el adverbio naka (dentro) y el verbo imasu (estar).

(5)

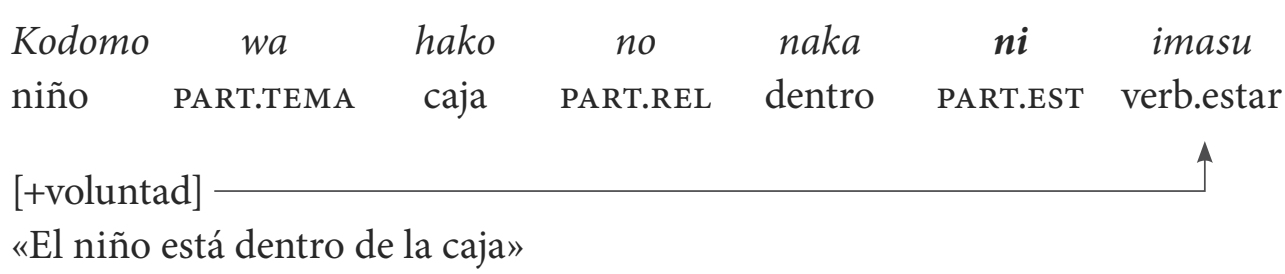

\section{Formalización}

De acuerdo con lo establecido en la semántica formal, emplearemos el símbolo sigma para representar la relación atributiva de las entidades con el lugar o propiedad. De modo que la oración 5 se representa del siguiente modo: $\sum$ naka (Kodomo, hako) donde naka es un adverbio que expresa una localización interior.

Gráfico 5. Diagrama arbóreo de la estructura semántica del estado locativo en la oración Kodomo wa hako no naka ni imasu

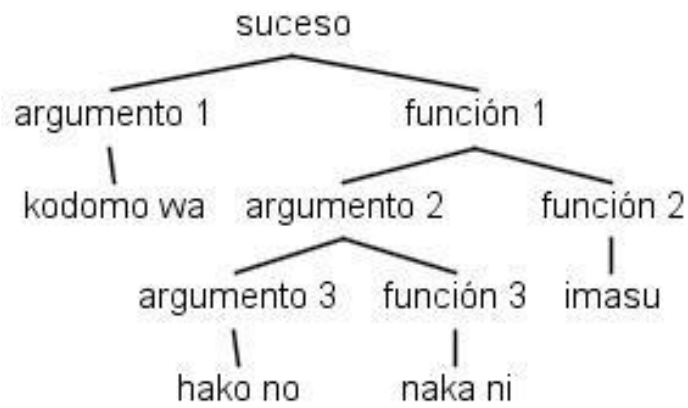

188 Lengua y Sociedad. Revista de Lingüística Teórica y Aplicada 
Análisis de la partícula japonesa ni en oraciones que expresan estados locativos Andrea Rodríguez Bravo y Claudia Ximena Garay Ramírez

Como se observa en el gráfico 5 , la partícula ni acompaña al adverbio naka (dentro) no aporta un significado, por lo tanto, cumple solo una función estructural, ya que se presenta de manera anterior al verbo imasu (estar).

En (6) se presenta una relación de localización, entre las entidades ringo (manzana) y hon (libro). Específicamente se presenta una relación supresiva, puesto que la manzana se posiciona ue (arriba) de hon (libro). En la oración aparece el verbo arimasu porque ringo (manzana) es un ser sin voluntad. Además, se presenta la partícula wa encargada de marcar el sujeto de la oración ringo (manzana). Otra partícula con carga semántica que se presenta es no la cual expresa que existe una relación entre ringo (manzana) y hon (libro). En cuanto a la partícula $n i$, esta se presenta entre el adverbio ue (arriba) y el verbo arimasu (estar).

(6)

$\begin{array}{lcccccc}\text { Ringo } & \text { wa } & \text { hon } & \text { no } & \text { ue } & \text { ni } & \text { arimasu } \\ \text { manzana } & \text { PART.TEMA } & \text { libro } & \text { PART.REL } & \text { arriba } & \text { PART.EST } & \text { verb.estar }\end{array}$

[-voluntad]

«La manzana está encima del libro»

\section{Formalización}

De acuerdo con lo establecido en la semántica formal, emplearemos el símbolo sigma para representar la relación atributiva de las entidades con el lugar o propiedad. De modo que la oración 6 se representa del siguiente modo: $\sum$ ue (Ringo, hon) donde ue es un adverbio que expresa una localización supresiva.

\section{Gráfico 6. Diagrama arbóreo de la estructura semántica del estado} locativo en la oración Ringo wa hon no ue ni arimasu

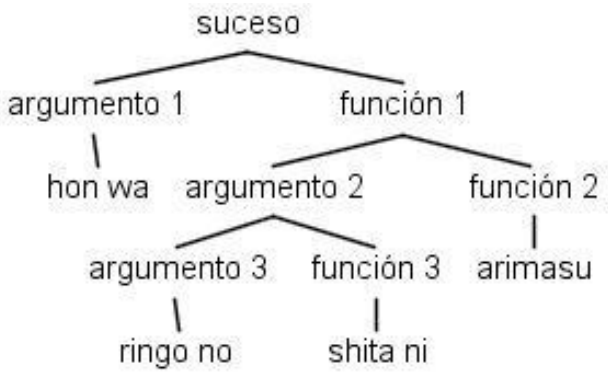


Análisis de la partícula japonesa ni en oraciones que expresan estados locativos Andrea Rodríguez Bravo y Claudia Ximena Garay Ramírez

Como se observa en el gráfico 6, la partícula ni acompaña al adverbio ue (dentro) no aporta un significado, por lo tanto, cumple solo una función estructural, ya que se presenta de manera anterior al verbo arimasu (estar).

En (7) se presenta una relación de localización, entre las entidades hon (libro) y ringo (manzana). Específicamente se presenta una relación subesiva, puesto que el libro se posiciona shita (abajo) de ringo (manzana). En la oración aparece el verbo arimasu porque hon (libro) es un ser sin voluntad. Además, se presenta la partícula wa encargada de marcar el sujeto de la oración hon (libro). Otra partícula con carga semántica que se presenta es no la cual expresa que existe una relación entre hon (libro) y ringo (manzana). En cuanto a la partícula ni, esta se presenta entre el adverbio shita (abajo) y el verbo arimasu (estar).

(7)

\begin{tabular}{|c|c|c|c|c|c|c|}
\hline Hon & $w a$ & ringo & no & shita & $n i$ & arimasu \\
\hline libro & PART.TEMA & manzana & PART.TEMA & abajo & PART.EST & verb.estar \\
\hline
\end{tabular}

\section{Formalización}

De acuerdo con lo establecido en la semántica formal, emplearemos el símbolo sigma para representar la relación atributiva de las entidades con el lugar o propiedad. De modo que la oración 7 se representa del siguiente modo: $\sum$ shita (Ringo, hon) donde shita es un adverbio que expresa una localización supresiva.

\section{Gráfico 7. Diagrama arbóreo de la estructura semántica del estado locativo en la oración Hon wa ringo no shita ni arimasu}

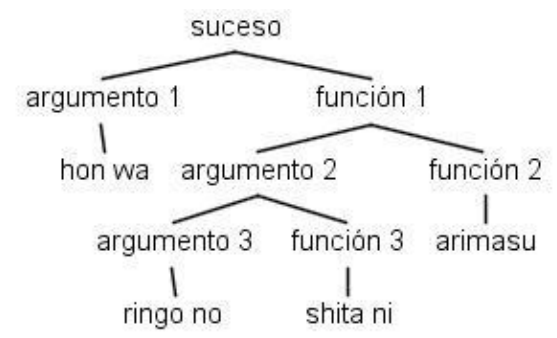


Análisis de la partícula japonesa ni en oraciones que expresan estados locativos Andrea Rodríguez Bravo y Claudia Ximena Garay Ramírez

Como se observa en el gráfico 7 , la partícula $n i$ acompaña al adverbio shita (abajo) no aporta un significado, por lo tanto, cumple solo una función estructural, ya que se presenta de manera anterior al verbo arimasu (estar).

En (8) se presenta una relación de localización, entre las entidades supuun (cuchara) y teberu (mesa). Específicamente se presenta una relación orativa, puesto que que la cuchara se posiciona sumikko (al borde) de teberu (mesa). En la oración aparece el verbo arimasu porque supuun (cuchara) es un ser sin voluntad. Además, se presenta no la cual expresa que existe una relación entre supuun (cuchara) y teberu (mesa). En cuanto a la partícula ni, esta se presenta entre el adverbio sumikko (al borde) y el verbo arimasu (estar).

(8)

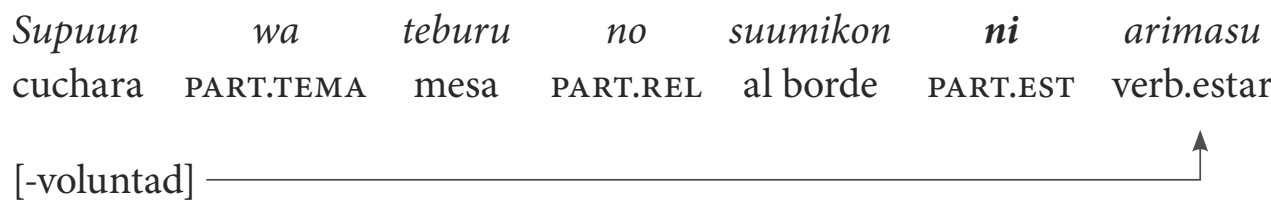

«La cuchara está al borde de la mesa»

\section{Formalización}

De acuerdo con lo establecido en la semántica formal, emplearemos el símbolo sigma para representar la relación atributiva de las entidades con el lugar o propiedad. De modo que la oración 8 se representa del siguiente modo: $\sum$ sumikko (Supuun, teburu) donde sumikko es un adverbio que expresa una localización orativa.

Gráfico 8. Diagrama arbóreo de la estructura semántica del estado locativo en la oración Supuun wa teburu no suumikon ni arimasu

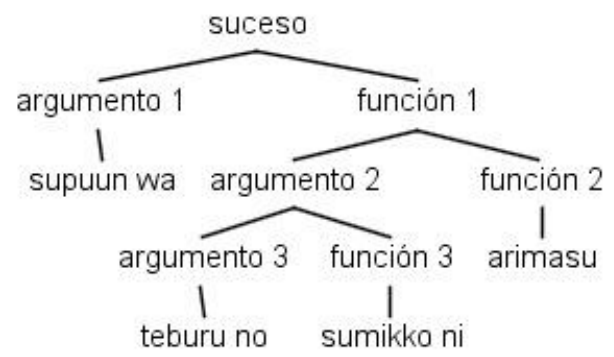


Análisis de la partícula japonesa ni en oraciones que expresan estados locativos Andrea Rodríguez Bravo y Claudia Ximena Garay Ramírez

Como se observa en el gráfico 8, la partícula ni acompaña al adverbio sumikko (al borde) no aporta un significado, por lo tanto, cumple solo una función estructural, ya que se presenta de manera anterior al verbo arimasu (estar).

En (9) se presenta una relación de localización, entre las entidades sara (plato) y hana (flor). Específicamente se presenta una relación medial, puesto que la flor se posiciona mannaka (al centro) de sara (plato). En la oración aparece el verbo arimasu porque hana (flor) es un ser sin voluntad. Además, se presenta la partícula wa encargada de marcar el sujeto de la oración hana (flor). Otra partícula que se presenta es ga esta cumple una función meramente estructural ya que no aporta algún significado en la oración. En cuanto a la partícula ni, esta se presenta entre el adverbio mannaka (al centro) y el verbo arimasu (haber).

(9)

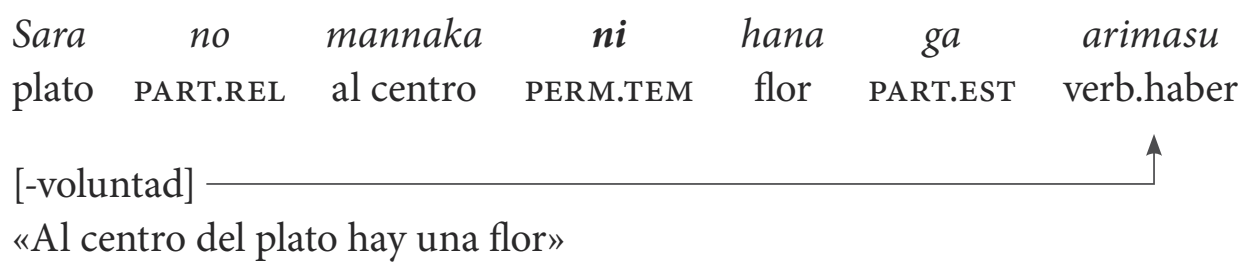

\section{Formalización}

De acuerdo con lo establecido en la semántica formal, emplearemos el símbolo sigma para representar la relación atributiva de las entidades con el lugar o propiedad. De modo que la oración 9 se representa del siguiente modo: $\sum$ mannaka (Hana, sara) donde mannaka es un adverbio que expresa una localización medial.

Gráfico 9. Diagrama arbóreo de la estructura semántica del estado locativo en la oración Sara no mannaka ni hana ga arimasu

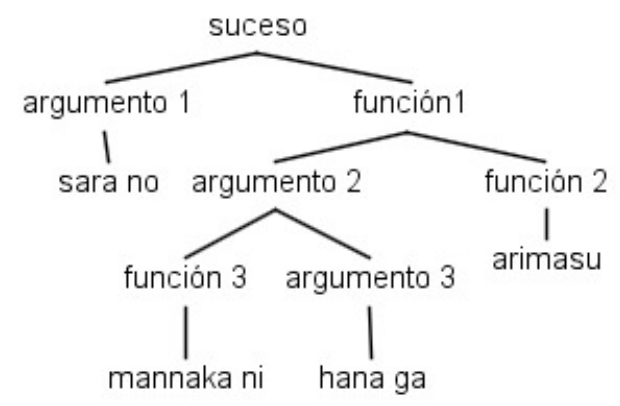

192 Lengua y Sociedad. Revista de Lingüística Teórica y Aplicada 
Análisis de la partícula japonesa ni en oraciones que expresan estados locativos Andrea Rodríguez Bravo y Claudia Ximena Garay Ramírez

Como se observa en el gráfico 9, la partícula ni acompaña al adverbio mannaka (al centro) aportando un significado, por lo tanto, cumple solo una función semántica, ya que se presenta de manera anterior al sustantivo hana (flor).

\section{Resultados y Discusión}

A partir del análisis observamos que las oraciones simples que expresan estados locativos - excesivos e inesivos - no presentaron diferencias en cuanto al uso de las partículas, ni en el orden estructural de la oración. A continuación, se presenta un cuadro con las partículas en japonesas usadas en el corpus, donde se muestra su significación o uso.

Tabla 1. Partículas en japonés

\begin{tabular}{|c|c|c|c|c|c|}
\hline Partículas & $w a$ & no & \multicolumn{2}{|c|}{$n i$} & $g a$ \\
\hline \multirow[t]{2}{*}{ 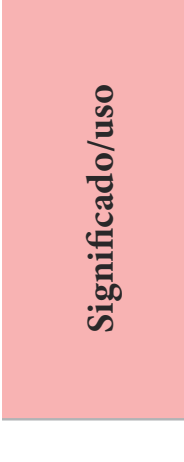 } & $\begin{array}{l}\text { Marca el } \\
\text { tema en la } \\
\text { oración. }\end{array}$ & $\begin{array}{l}\text { Partícula } \\
\text { que } \\
\text { relaciona } \\
\text { o ubica } \\
\text { objetos entre } \\
\text { sí; sujeto y } \\
\text { objeto }\end{array}$ & $\begin{array}{l}\text { Marcar el } \\
\text { lugar de } \\
\text { una acción } \\
\text { que expresa } \\
\text { permanencia } \\
\text { temporal. }\end{array}$ & $\begin{array}{l}\text { Cuando } \\
\text { a este le } \\
\text { antecede un } \\
\text { adverbio y } \\
\text { le precede } \\
\text { un verbo de } \\
\text { existencia: } \\
\text { arimasu o } \\
\text { imasu. }\end{array}$ & $\begin{array}{l}\text { Cuando } \\
\text { a este le } \\
\text { antecede un } \\
\text { nombre y } \\
\text { le precede } \\
\text { un verbo de } \\
\text { existencia: } \\
\text { arimasu o } \\
\text { imasu. }\end{array}$ \\
\hline & \multicolumn{3}{|c|}{ Función semántica } & \multicolumn{2}{|c|}{ Función estructural } \\
\hline
\end{tabular}

Como se observa en el corpus se presentan dos partículas estructurales: $n i$ y $g a$, que oscilan. Ambas partículas se presentan de manera anterior al verbo de la oración, la deferencia de una u otra radica en que la primera se presenta después del adverbio, y la segunda se presenta después de un nombre, respectivamente.

Las partículas con carga semántica del presente corpus son tres: La primera es $w a$, esta remite al tema o tópico oracional (Imaeda, Kumano, Suzuki \& Fujino, 2012); en otras palabras, aquel del cual se hablará en la oración. La segunda es no, la cual aporta el significado de relación entre dos objetos o un objeto y un sujeto. La tercera es ni, contribuye aportando el significado de permanencia temporal.

Por otro lado, se presenta una oscilación entre los verbos arimasu e imasu (equivalentes al verbo estar). La existencia de estos verbos en la oración se explica 
Análisis de la partícula japonesa ni en oraciones que expresan estados locativos Andrea Rodríguez Bravo y Claudia Ximena Garay Ramírez

si en esta se incluye un tópico inanimado (sin voluntad) se presenta el primer verbo, y de ser un tópico animado (con voluntad) se presentará el segundo verbo. A continuación, se presenta un cuadro que explica la oscilación entre los verbos arimasu e imasu.

Tabla 2. Alternancia de la posición del adverbio en las oraciones locativas

\begin{tabular}{l|l}
\hline Verbos & Uso \\
\hline Arimasu & $\begin{array}{l}\text { Cuando referimos a la } \\
\text { presencia de sujetos } \\
\text { oracionales inanimadas. }\end{array}$ \\
\hline Imasu & $\begin{array}{l}\text { Cuando referimos a la } \\
\text { presencia de sujetos } \\
\text { oracionales animados. }\end{array}$ \\
\hline
\end{tabular}

\section{Conclusión}

La relación que se estable entre las entidades de las oraciones presentadas en el corpus es determinada por los adverbios, comprobando de esta manera los adjetivos, adverbios y preposiciones denotan este tipo de relaciones atemporales según Langacker (1986, como se citó en Moreno, 2003). Este autor menciona que las relaciones temporales pueden presentarse en la oración; sin embargo, no desempeñan un papel central. Entonces, aunque aparezca la partícula ni (marca de temporalidad) la relación locativa sigue siendo la determinante en la oración. Además, este trabajo da cuenta de la relación entre la semántica y la sintaxis, tal como describe la teoría de la semántica formal; puesto que, la inserción u omisión de la partícula wa modifica el orden sintáctico de la oración. Por otro lado, cabe mencionar que independiente de presentarse una localización inesiva o excesiva, la partícula $n i$ puede aportar carga semántica o cumplir solo una función estructural.

194 Lengua y Sociedad. Revista de Lingüística Teórica y Aplicada 
Análisis de la partícula japonesa ni en oraciones que expresan estados locativos Andrea Rodríguez Bravo y Claudia Ximena Garay Ramírez

\section{Financiamiento}

La investigación se realizó sin financiamiento.

Conflicto de intereses.

Las autoras no presentan conflicto de interés

\section{Agradecimientos}

Las autoras agradecen los alcances brindados por la lingüista Verónica Lazo García, profesora del curso de Semántica in en la Universidad Nacional Mayor de San Marcos.

\section{Referencias bibliográficas}

Cardona, J. (2020). Nociones básicas sobre el idioma japonés. Guía para hispanohablantes. Recuperado de: http://nihongo.monash.edu/jp_gram_esp.pdf

Cipris, Z. \& Hamano, S. (2002). Making Sense of Japanese Grammar: A Clear Guide through Common Problems. University of Hawai'i Press. pp. 25-28. https://bit.ly/2Zxk2dw

Cook, H. (1999). Particles. Journal of Linguistic Anthropology. 181-183. https:// bit.ly/3BuaayC

Endo, Y. (2007) Locality and Information Structure: A Cartographic Approach to Japanese. John Benjamins.

Gonzales, V. \& Isshiki, T. (1986). Diccionario Español-Japonés (Seiwa Jiten).

Imaeda, A., Kumano, N., Suzuki, Y., Fujino, H. (2012). Marugoto Gramática A1. https://www.fundacionjapon.es/userfiles/file/Curso\%20JFs/Marugoto\%20 Gramatica\%2oEs.pdf

Iori, I., Takanashi, N., Nakanis hi, K., Yamada, T. (2000, 2010) Shokyu-wo oshieru hito-no tame-no nihongo bunpou handobukku (El manual de la gramática japonesa para los que dan clases de nivel elemental). Suri e netto wakku.

Kishi, Daisuke (2015). El uso de las partículas japonesas en el Arte de la lengua japona, de Melchor Oyanguren. México y la Cuenca del Pacífico. (12), 93-114. https://www.redalyc.org/articulo.oa?id=4337/433747302006

Kuno, S. (1972) 'Functional Sentence Perspective: A Case Study from Japanese and English.' Linguistic Inquiry, 3(3). https://www.jstor.org/stable/4177715

Hirano, T. (2016). Entendiendo las partículas $\nabla$ (wa) y $\nabla$ (ga). Espai wabi-sabi. Recuperado de: https://www.espaiwabisabi.com/ particulas-wa-y-ga/?v=3acf83834396 
Análisis de la partícula japonesa ni en oraciones que expresan estados locativos Andrea Rodríguez Bravo y Claudia Ximena Garay Ramírez

Hirano, T. (2016). Utilizar correctamente las partículas $\bigotimes(n i) y \bigotimes($ de). Espai wabisabi. https://www.espaiwabisabi.com/particulas-ni-y-de/?v=3acf83834396

Mio, I. (1948) Kokugo-bunpo-shouron (Una compilación de documentos sobre la gramática de la lengua japonesa). Sanseidou.

Moreno, C. (2003). Sucesos, papeles semánticos y relaciones sintácticas. Madrid. Ed. A. Manchado Libros.

Nishu, M. (2015) Usos correctos de las partículas en japonés: una reseña sobre las partículas de caso. Open Journal Systems. (4), 171-185. https://ojs.ehu. eus/index.php/Mundo/article/view/14822 Porta, L. \& Matsuura, J. (2021). Diccionario Pocket Japonés.

Rivera, H. (2017). La partícula «囚». Japolatino. Recuperado de: http://www.kimisikita.org/gram2/particno.html

Santos, L. (2020). Fundamentos para una semántica mínima (Tesis de doctorado) Universidad de Valladolid. España.

Senko K. Maynard. (2011). Learning Japanese for Real: A Guide to Grammar, Use, and Genres of the Nihongo World. University of Hawai'i Press. pp. 51-66. Recuperado de: https://bit.ly/3modnLK

Taranov, A. (2013). Vocabulario español-japonés. Blackwell.

Tsujim ura, N. (1996) An Introduction to Japanese Linguistics. Blackwell. 
Análisis de la partícula japonesa ni en oraciones que expresan estados locativos Andrea Rodríguez Bravo y Claudia Ximena Garay Ramírez

\section{Anexos}

anexo 1

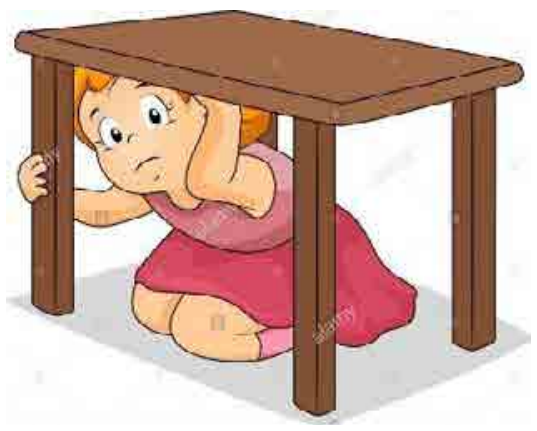

anexo 3

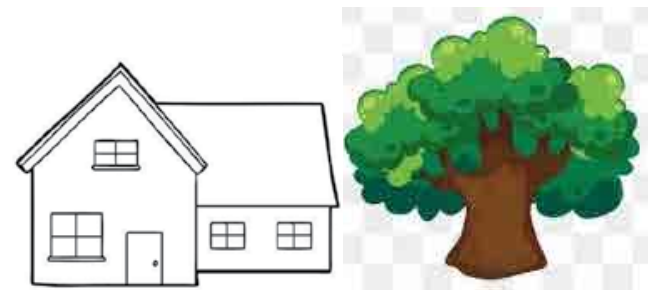

anexo 5

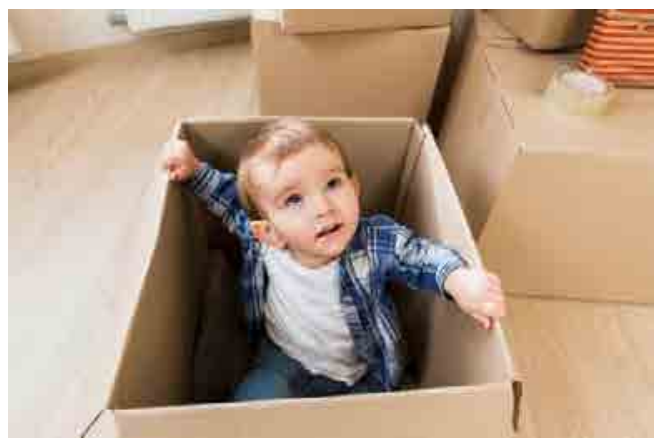

anexo 2

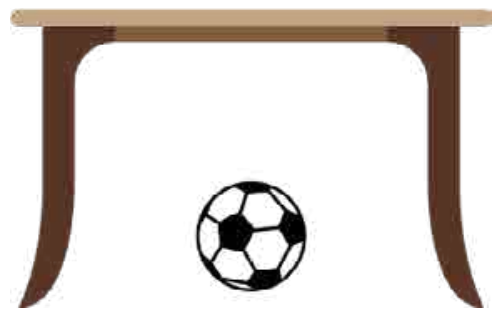

anexo 4

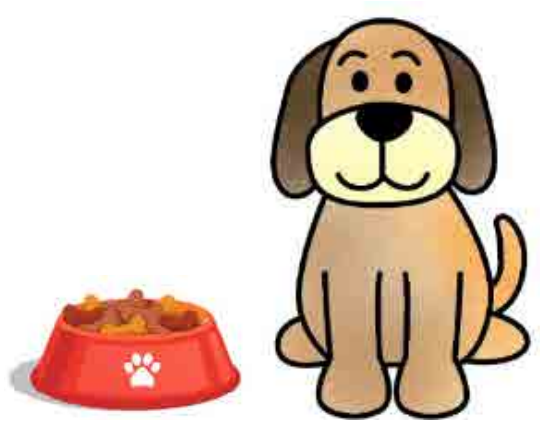

anexo 6

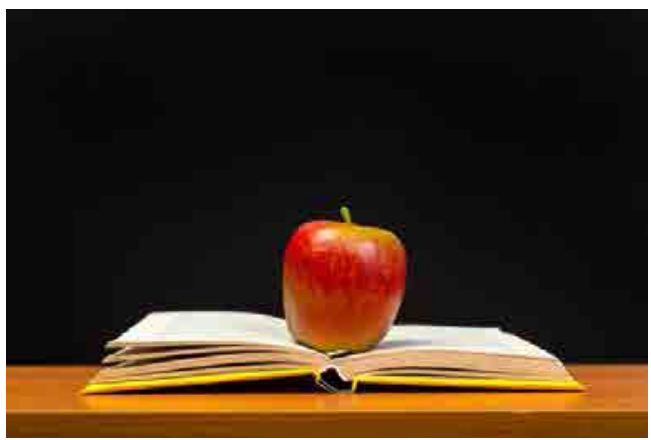


Análisis de la partícula japonesa ni en oraciones que expresan estados locativos Andrea Rodríguez Bravo y Claudia Ximena Garay Ramírez

anexo 7

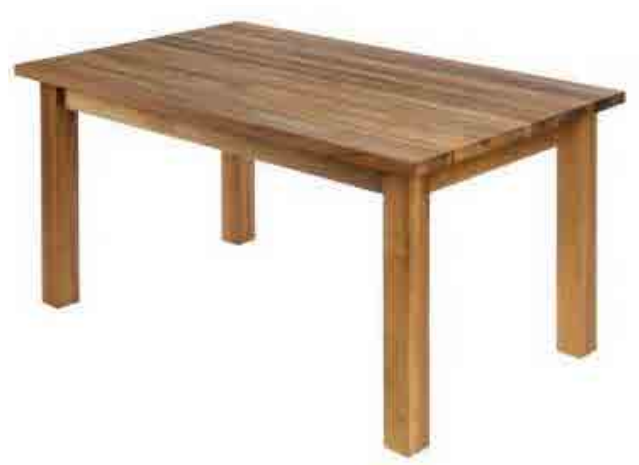

anexo 8

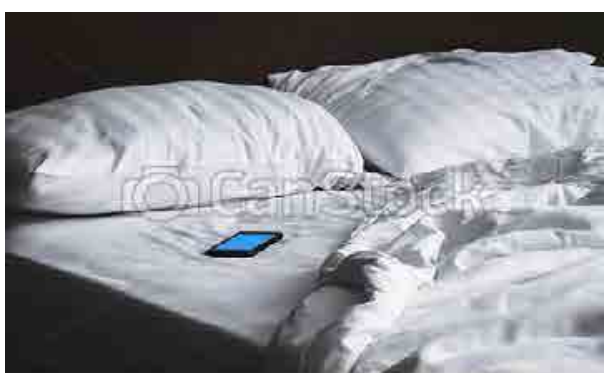

anexo 9

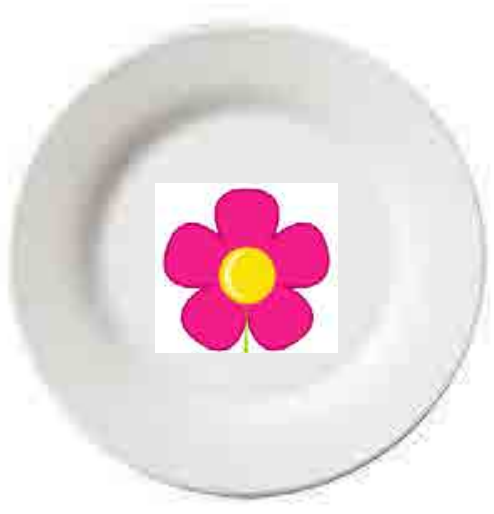

198 Lengua y Sociedad. Revista de Lingüística Teórica y Aplicada 
Análisis de la partícula japonesa ni en oraciones que expresan estados locativos Andrea Rodríguez Bravo y Claudia Ximena Garay Ramírez

\begin{tabular}{l|l}
\hline Abreviatura & Significado \\
\hline PART.REL & Partícula relacional \\
\hline PART. EST & Partícula estructural \\
\hline PART.TEM & Partícula temporal \\
\hline
\end{tabular}

\section{Trayectoria académica}

\section{Andrea Rodriguez Bravo}

Estudiante de lingüística en la Universidad Nacional Mayor de San Marcos (UNMSM), miembro adjunto del Gabinete Lingüística Forense. Sus áreas de interés son los estudios relacionados con la semántica, fonética y fonología. Actualmente, labora como asistente en lingüística en el Ministerio Público.

\section{Ximena Garay Ramírez}

Estudiante de lingüística en la Universidad Nacional Mayor de San Marcos (UnMSM), miembro del grupo de investigación Lenguas y Filosofías del Perú (LFP). Sus áreas de interés son los estudios relacionados con la semántica, lexicografía y sociolingüística. Actualmente, labora como maestra de Educación Básica Regular (EBR). 